\title{
Approche historique de l'agriculture urbaine au Dahomey (Bénin)
}

Historical approach to urban agriculture in Dahomey (Benin)

\section{Dominique Juhé-Beaulaton}

\section{(2) OpenEdition}

\section{Journals}

Édition électronique

URL : http://journals.openedition.org/ethnoecologie/2296

DOI : $10.4000 /$ ethnoecologie.2296

ISSN : 2267-2419

Éditeur

Laboratoire Eco-anthropologie et Ethnobiologie

Référence électronique

Dominique Juhé-Beaulaton, «Approche historique de l'agriculture urbaine au Dahomey (Bénin) », Revue d'ethnoécologie [En ligne], 8 | 2015, mis en ligne le 31 décembre 2015, consulté le 01 mai 2019. URL : http://journals.openedition.org/ethnoecologie/2296; DOI : 10.4000/ethnoecologie.2296

Ce document a été généré automatiquement le 1 mai 2019.

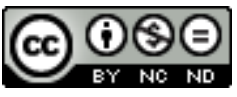

Revue d'ethnoécologie est mis à disposition selon les termes de la licence Creative Commons Attribution - Pas d'Utilisation Commerciale - Pas de Modification 4.0 International. 


\title{
Approche historique de l'agriculture urbaine au Dahomey (Bénin)
}

\author{
Historical approach to urban agriculture in Dahomey (Benin)
}

Dominique Juhé-Beaulaton

1 Si en Afrique la production agricole en périphérie urbaine est bien étudiée, l'histoire de ses origines et de son développement l'est beaucoup moins. Dans un contexte où l'agriculture en ville se développe dans les pays du nord, il est apparu opportun d'examiner cette situation dans un pays du sud qui permet à la fois de questionner la notion de ville et de resituer ce type de production dans la longue durée. Comme en bien d'autres pays, la croissance urbaine au Bénin, a jusqu'à récemment été surtout horizontale et a laissé la place à une production agricole « interstitielle », qu'il s'agisse du petit élevage - dont on ne traitera pas ici - ou de la culture de certaines plantes alimentaires, principalement fruitières, dans les cours des maisons familiales, sur les trottoirs ou tout autre espace considéré comme disponible. Cette production agricole en ville venait en complément de l'alimentation dite de base, avec essentiellement des céréales et des tubercules accompagnés de plantes à sauce.

2 Le Bénin méridional, aire géographique de cette étude, était connu avant la colonisation sous le nom de Côte des Esclaves, située en Afrique occidentale, dans le golfe de Guinée, région où le domaine forestier guinéo-congolais s'interrompt pour laisser place à des paysages de savanes et de champs cultivés (Figure 1). Les différents peuples de cette région appartiennent pour la majorité d'entre eux à une même aire culturelle ${ }^{1}$, dénommée Aja-Tado ou Aja-Fon (Gayibor 1985). À partir du XVII siècle, la région a connu l'essor de plusieurs royaumes, dont ceux de Ouidah, Allada, Porto Novo et le plus connu, le Dahomey qui imposa au XVIII ${ }^{\mathrm{e}}$ siècle sa domination sur les autres royaumes jusqu'à sa défaite lors de la conquête coloniale française en 1894. Le processus d'urbanisation est relativement ancien, organisé d'une part autour des cours royales et de leur palais, et d'autre part autour des forts européens implantés sur la côte ouest africaine en vue d'y développer le commerce, notamment celui des esclaves. Les Européens ont très tôt décrit les centres urbains et mis l'accent sur la densité de la population de la région, aujourd'hui une des plus fortes d'Afrique, variant entre 200 à 400 habitants au km². 
Figure 1 : Localisation de l'étude : royaumes de l'aire culturelle aja-tado



3 Les sources historiques à notre disposition pour comprendre l'évolution dans le temps des systèmes de production agricole sont de nature variée. Elles proviennent en grande partie des documents écrits : des récits de voyages et des documents d'archives rédigés par les navigateurs, les résidents des forts européens, des missionnaires, des commerçants et des explorateurs présents sur cette côte du XVII ${ }^{\mathrm{e}}$ au XIX $\mathrm{X}^{\mathrm{e}}$ siècles, ainsi que des rapports des premiers administrateurs coloniaux. Des informations orales recueillies au cours d'enquêtes de terrain de 1990 à 2008 complètent la documentation ${ }^{2}$. Le croisement de ces sources permet de se faire une idée de la place et de l'organisation de l'agriculture dans les villes du Dahomey, d'en saisir les évolutions sous diverses influences liées à la présence européenne, à la nature du commerce pratiqué et des pouvoirs politiques dominants, et de constater le rôle éminent des centres urbains dans les mutations agricoles, du XVII ${ }^{\mathrm{e}}$ siècle jusqu'à l'instauration du pouvoir colonial au début du $\mathrm{xx}^{\mathrm{e}}$ siècle.

\section{Organisation de l'espace : ville, village, campement de cultures}

4 Selon les pays, des critères différents sont pris en compte pour définir la ville ; en France, comme en Espagne ou en Italie, c'est le franchissement d'un seuil de population qui la caractérise; elle correspond à une agglomération relativement importante, dont les habitants ont des activités professionnelles diversifiées. La densité du bâti autour d'un centre peut être prise en compte, ou ses fonctions administratives et commerciales, mais il s'avère que chaque pays a sa propre conception, ce qui limite les comparaisons. Des points communs rapprochent les villes européennes établies autour d'un vieux centre et 
évoluant selon une croissance concentrique qui dépasse les limites dans lesquelles les villes étaient autrefois enfermées (Le Gleau et al. 1996).

Les sources historiques nous apprennent que dans la région étudiée, avant la conquête coloniale de la fin du $\mathrm{XIX}^{\mathrm{e}}$ siècle, le tissu urbain était lâche, peu dense et la place du végétal importante. Les voyageurs européens ont perçu les établissements humains à travers leur propre système de représentation et ils ont ainsi qualifié de villages des villes souvent très peuplées, car elles pouvaient atteindre plusieurs milliers d'habitants, mais incluaient cependant des zones de cultures qui renforçaient l'impression de ruralité ${ }^{3}$. Ils ont également été frappés par le réseau des voies de communication, relatant l'irrégularité des rues. L'administrateur d'Albéca (1895: 126) a bien décrit «le dédale inextricable de sentiers et d'impasses " dans lequel les Européens se perdaient et il a dénoncé l'insalubrité des villes en saison des pluies. Toutes les maisons, basses et couvertes de chaume, étaient entourées d'un mur de terre, formant un enclos, appelé aussi concession (en français colonial), terme encore utilisé aujourd'hui. Des arbres forestiers et fruitiers, dont des orangers et des bananiers, y poussaient et les Européens, à la vue de ces espaces arborés, éprouvaient quelque difficulté à s'imaginer dans la capitale d'un grand pays (Forbes 1851). Ces éléments caractérisent toujours nombre de villes du Bénin, ce qui amène à réfléchir à la notion de ville, tant les limites apparaissent floues.

6 Si le terme « ville » ne semblait pas correspondre à l'idée que s'en faisaient les auteurs des $\mathrm{XVIII}^{\mathrm{e}}$ et XIX ${ }^{\mathrm{e}}$ siècles, ils l'appliquaient néanmoins à ce qu'ils observaient ${ }^{4}$. L'expression de «centre habité » est peut-être plus appropriée pour désigner ces "agglomérations humaines » que les termes « ville» ou « village » utilisés dans les sources anciennes. Un voyageur hollandais visitant le royaume de Ouidah à la fin du XVII siècle distingua les grands villages des petits et précisa que les terres étaient semées jusque dans les haies qui entourent les maisons (Bosman 1705 : 356-357).

7 L'historien du Dahomey, R. Law, définit l'urbanité plus par l'autonomie politique et le rôle administratif de certaines agglomérations que par la concentration démographique en un lieu (Law 2004). Et, de fait, nous allons voir avec plusieurs villes de cet ancien royaume, Ouidah, Abomey, Cana, que des caractéristiques communes se dégageaient dans leur organisation spatiale et agronomique, même si leurs fonctions semblaient les opposer.

\section{Des fonctions différentes}

8 En effet, si la ville côtière de Ouidah s'est développée autour de l'activité commerciale avec les Européens, Cana et Abomey, anciennes capitales des rois du Dahomey, sont les mieux connues des « cités-palais $»^{5}$ situées à 150 kilomètres au nord dans l'intérieur des terres. La traite des esclaves a fait la richesse de la ville côtière de Ouidah et attisé les convoitises du royaume du Dahomey situé au nord. En 1727, le roi du Dahomey finit par conquérir Ouidah; il institua un vice-roi en 1733 et y installa une garnison militaire. La ville connut alors un véritable essor à la fois spatial - elle comprenait six quartiers - et commercial qui lui donna un aspect multi-centres différent de celui des villes royales comme Abomey où le palais du roi régnant représentait le centre de l'agglomération. Ouidah constituait le pôle commercial du royaume alors qu'Abomey combinait les fonctions de siège de l'administration centrale, de lieu de célébration des cultes royaux et de pôle commercial. Ouidah devint ainsi un centre beaucoup plus important à la fois sur le plan commercial, mais aussi comme pôle "provincial» de l'administration dahoméenne très centralisée autour de la personne royale et de sa capitale principale, 
Abomey, où les troupes d'élite résidaient ainsi que les dignitaires chargés des principaux offices, souvent comparés à des ministres par les Européens. Les cultivateurs y apportaient chaque année les prémices de leurs récoltes, dont une partie était gardée dans les greniers du roi qui garantissaient ainsi une meilleure protection des semences stockées contre les incendies. À la fin du XVIII ${ }^{\mathrm{e}}$ siècle, les Européens y étaient invités chaque année pour assister aux cérémonies dédiées aux ancêtres royaux ${ }^{6}$, accompagnées de sacrifices rituels et de banquets, occasions de dons entre les autorités dahoméennes et les représentants européens parmi lesquels figuraient des plantes, comme l'igname donnée aux voyageurs, ou le piment d'origine américaine offert au roi par un gouverneur.

\section{Mais une exploitation agricole comparable}

Quelles que soient leurs fonctions, les villes du Dahomey disposaient d'espaces dédiés à la culture et/ou aux arbres. La ville d'Abomey se distinguait par ses hautes murailles en terre doublées d'un fossé. Cette fortification d'un périmètre d'une dizaine de kilomètres renfermait les palais de plusieurs rois, des terres cultivées et des friches, comme l'ont rapporté certains auteurs, dont Frederick Forbes, un officier de la marine britannique (Forbes 1851: 70) ; le lieutenant de vaisseau français Auguste Bouët mentionne «une vaste étendue de terrain à cause des jardins et des enclos $»^{7}$. La ville, d'après lui, atteignait 30000 habitants et devait se défendre des incursions de ses voisins yoroubas ${ }^{8}$. À Ouidah, il n'y avait pas de fortifications, mais les forts construits par les Européens favorisèrent le développement de la ville en parallèle avec l'essor de la traite des esclaves - Ouidah étant un des principaux ports d'exportation des captifs jusqu'à la seconde moitié du XIX siècle -. Les activités agricoles y avaient néanmoins leur place dans l'économie urbaine, comme à Abomey. La première dénomination de Ouidah, Glexwe, signifiait d'ailleurs «maison des champs ». Au XVIII siècle, la ville de Ouidah comportait déjà des espaces ouverts cultivés (Bosman 1705: 356 ; Law 2004: 79). L'agriculture reposait essentiellement sur le travail des femmes et des esclaves (qui n'étaient pas tous destinés à la traite). Le docteur Repin, ancien chirurgien de la marine, relatait que Ouidah « comme toutes les villes nègres, [...] occup[ait] un espace de terrain considérable à cause des nombreux groupes d'arbres magnifiques et des jardins très étendus qu'elle renferme[ait]»(Repin 1863: 70). Le diplomate et explorateur R.Burton indiquait qu'il y avait plus de brousse que d'habitations, sauf autour du marché (1864: 60). C'est comme la capitale [Abomey], « une agglomération de villages » (Burton 1864: 64). De même, selon l'administrateur colonial Foa (1895) :

"La végétation se développe librement; dans tous les endroits sans construction et dans l'enclos de chaque case, il y a quelques arbres ou arbustes. On rencontre ainsi par la ville, et semés avec une irrégularité artistique, des manguiers, des bananiers, des cocotiers, des corossols, des mimosées, des acacias multicolores, encombrés de plantes grimpantes qui ajoutent leur feuillage capricieux aux enclos élevés par l'homme [...] il y a des rues qui semblent des allées de bosquet, des cases qui sont des nids de feuillage. [...] Les divinités fétiches, semées çà et là, se détachent comme des statues mal ébauchées, de ce cadre de verdure. [...] On voit peu d'endroits sans végétation; il est rare que la nature ne fasse pas à chaque recoin de petits jardins à sa fantaisie. [...] à Ouidah, que les Européens surnommèrent le Jardin du Dahomey, des milliers d'orangers et de citronniers répandent, au temps de la floraison, leur suave parfum par la ville... » (Foa 1895 : 412) 
Cette description reprend les caractéristiques de l'ensemble des descriptions de villes, marquées par la présence d'une végétation luxuriante, d'apparence inorganisée, restée indéterminée et dominée par des arbres fruitiers plus faciles à identifier.

\section{Une occupation des villes variable dans le temps}

11 Certains voyageurs ont observé que les cités-palais n'étaient pas occupées de façon permanente par la cour royale. Le lieutenant de vaisseau Vallon décrivant la ville de Cana formée d'une "agglomération de villages séparés les uns des autres par de véritables jardins entourés de murs en terre ou de haies vives » précisa par exemple que le « roi y fix [ait] la résidence de la cour à l'époque des fêtes religieuses.»(Vallon 1861: 336). L'administrateur d'Albéca souligna également, près de quarante ans après le passage de Vallon, que Cana, où « les rois [y] avaient une résidence entourée de hautes murailles, et les ministres leur maison de campagne" restaient «inoccupées une grande partie de l'année » (Albéca 1895 : 103).

«Cana ancienne capitale du Dahomey [...].

12 Après la conquête coloniale de 1894 , Cana perdit son statut de ville royale et n'était " plus qu'une agglomération de cases en pisé au milieu de jardins, de cultures et d'arbres fruitiers» (Albéca 1895: 103), mais elle conserva cependant un rôle religieux lié à la proximité d'une source sacrée dont l'eau intervenait dans les rituels d'intronisation et de purification des rois du Dahomey.

13 En 1895, Albéca nota que l'occupation de ces centres habités variait dans le temps, en fonction des déplacements du roi et de sa cour mais aussi des saisons agricoles. D'après cet auteur, les modifications dans l'extension des villes et la densité de leur population dépendaient de leur fonction ou des événements et cette organisation se retrouvait dans toutes les villes (Albéca $1895: 126$ ).

14 Les informations orales que j'ai pu recueillir en complément de ces textes révèlent que les agriculteurs résidaient en un centre habité et le quittaient selon les saisons pour demeurer dans leurs champs plus ou moins éloignés; les pratiques agricoles imposaient une culture sur brûlis après défrichement, une rotation des cultures et un déplacement des parcelles lorsque la terre n'était plus assez fertile. Ils effectuaient donc des allersretours entre les campements de cultures, occupés au moment de la préparation des champs et des récoltes, et les centres habités, lieux d'échanges autour de marchés qui constituaient des pôles de rassemblement pour les habitants. Il n'existait donc pas de limite clairement définie entre milieu urbain et rural mais plutôt un continuum pouvant lui-même varier en fonction des mouvements de population liés aux saisons, aux périodes de cérémonies, aux épisodes guerriers et aux relations avec les autres villes et populations. Ce qui reste stable, tout au moins jusqu'à la période coloniale, c'est la présence des cultures dans les espaces habités. La composition floristique de cette végétation arborée, d'après les auteurs anciens, accordait une place prépondérante aux espèces fruitières et aux palmiers, cocotiers ou palmiers à huile. Si les enclos familiaux comprennent des arbres et arbustes, il en est de même pour les espaces libres situés entre les concessions familiales bien délimitées dans l'espace par des haies ou des clôtures, et cette végétation formait des jardins vergers dont les contours, par contre, ne semblaient pas clairement définis. 


\section{La ville : un lieu d'innovation et de conservation}

\section{souvent dissimulés par une ceinture arborée. Palmiers, cocotiers et bananiers se} trouvaient à proximité et dans les villages. Ces centres se distinguaient au milieu des champs « par les frondaisons des vergers ou des arbres d'agrément conservés ou plantés pour l'ombrage » (Toutée 1897). Parmi ces « arbres de village » se trouvaient des fruitiers : citronniers, papayers, manguiers, cocotiers, bananiers, baobabs. Ces «vergers» ou "jardins $»^{9}$ signalaient donc aux observateurs étrangers l'existence d'une zone habitée. Ces deux termes ne sont plus guère employés dans la littérature contemporaine qui parle plutôt d'agroforesterie urbaine et péri-urbaine. En Guinée, les travaux de J. Fairhead et M. Leach (1996) ont parfaitement montré le développement parallèle de ces agroforêts villageoises et des établissements humains en zone de savane, jusqu'à constituer de véritables formations forestières périphériques. Au Bénin, le processus est bien décrit par les auteurs anciens, mais ces ceintures agroforestières apparaissent moins facilement aujourd'hui dans les paysages pourtant ouverts, en raison de la dispersion de l'habitat rural et de la forte densité démographique. On observe pourtant toujours un continuum urbain/rural s'accompagnant d'une densification des éléments arborés dans et autour des villes (Figure 2).

Figure 2 : Vue satellitaire de la ville d'Abomey

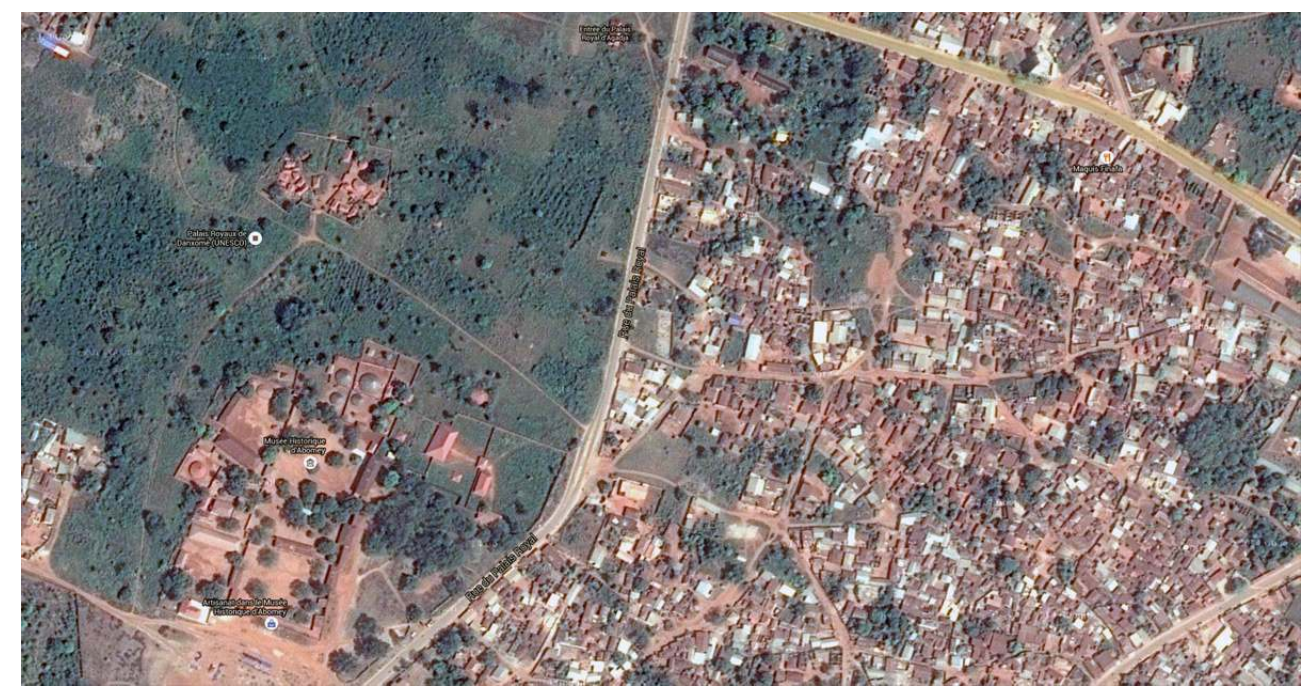

https://www.google.fr/maps/, mars 2015

Les arbres fruitiers plantés à proximité des habitations se retrouvent dans toute la zone étudiée ${ }^{10}$. Les espèces d'origine étrangère sont majoritaires, tels les agrumes, le manguier, le cocotier ou le corossolier. Notons que ces fruitiers étaient cités parmi les arbres villageois bien avant l'arrivée des missionnaires et des agronomes coloniaux. Dans la zone littorale (Grand Popo), certains auteurs ont établi une relation directe entre la fondation de nouveaux établissements et la plantation de cocotiers (Albéca 1895: 153). L'espace habité représentait alors la zone de prédilection des essais culturaux.

Néanmoins, un certain nombre d'arbres ou d'arbustes d'origine locale, comme le baobab, le palmier à huile, lissètin (Blighia sapida), sisrè (Synsepalum dulcificum) ou asrotin ( 
Irvingia gabonensis), y trouvaient leur place, simplement conservés mais parfois aussi plantés ${ }^{11}$. La présence d'un grand nombre de baobabs sur un site était considérée comme un indice favorable à l'installation des hommes, les pourvoyant abondamment en produits de cueillette, fruits et feuilles étant consommés. Lors d'une migration, les ressources immédiatement disponibles représentaient un facteur déterminant dans le choix de l'installation, au même titre que la fertilité des terres. Mais les habitants expliquent aussi la fréquence du baobab par l'occupation humaine, celle-ci favorisant le développement de certaines espèces par la consommation des fruits. Les textes anciens nous informent de la présence du palmier à huile à proximité immédiate des villes d'Abomey et de Ouidah, soit en dehors de sa zone écologique de prédilection; ceci peut être interprété également comme un indice de sa diffusion relativement récente (JuhéBeaulaton 1998 $)^{12}$. Au XVIII ${ }^{\mathrm{e}}$ siècle, les rois du Dahomey ont joué un rôle capital dans le développement des plantations de palmiers sur le plateau d'Abomey. Le géographe Henry Hubert observa d'ailleurs qu'ils se portaient mieux à l'intérieur des établissements humains, ce qu'il expliquait par la protection dont jouissaient les abords des agglomérations, particulièrement contre les feux de brousse (Hubert 1908: 130). Vers 1860, les voyageurs remarquèrent qu'un nombre incalculable de jeunes palmiers avaient été plantés autour des villes et des villages. Leur exploitation semblait se limiter à la périphérie des zones habitées, alors que les «forêts naturelles » auraient été capables de fournir une production abondante ; mais le manque de routes et de moyens de transport limitait leur exploitation. Les palmeraies cultivées se sont développées le long des routes commerciales et autour des centres habités, en l'occurrence les villes de Ouidah, Allada, Abomey, en relation avec les facilités d'exploitation et de transport vers les comptoirs et ports d'exportation.

Ces espaces urbains cultivés ne représentaient pas seulement des centres de domestication éventuelle d'espèces indigènes spontanées mais aussi des lieux d'intégration et de diffusion d'espèces étrangères. L'historien J. Vansina a déjà souligné en 1985 le rôle joué par les jardins ou les cultures proches des habitations en zone forestière dans l'introduction et la diffusion de nouvelles plantes dans les systèmes agricoles africains (Vansina 1985). Les villes apparaissent comme des lieux d'innovation dans le domaine agricole.

\section{Les premiers jardins européens et le développement du maraîchage}

Dès les premiers voyages au long cours, les navigateurs choisirent sur les côtes fréquentées des lieux favorables à l'approvisionnement en eau, bois, denrées alimentaires et au commerce avec les habitants. Certaines de ces escales devinrent des postes fixes, simples comptoirs ou établissements fortifiés, dont les jardins assuraient la production de vivres frais aux résidents permanents, aux membres des équipages lors des escales et aux esclaves qui y transitaient avant leur départ vers les Amériques (Figure 3). Le nombre d'orangers signalé plus haut par Foa (1895) est à mettre en relation avec la traite des esclaves et la nécessité de développer cette culture contribuant notamment à la lutte contre le scorbut qui ravageait les équipages et les captifs. Les auteurs étudiés ont mentionné des jardins dans les forts anglais, portugais et français de Ouidah. La liste des plantes cultivées dans ces jardins révèle la présence d'espèces maraîchères comme les choux, carottes, salades... introduites par les Européens peu après leur installation sur la 
côte et avant l'arrivée des missionnaires chrétiens vers $1830^{13}$. Ces jardins des forts et comptoirs fonctionnaient aussi en réseau d'échanges avec les autres forts de la côte ouest africaine, particulièrement ceux d'El Mina et de Cape Coast. Ils ont ainsi servi à l'acclimatation d'un grand nombre de plantes aujourd'hui passées dans les systèmes agricoles locaux. La ville de Ouidah, rappelons-le, était organisée en différents quartiers autour de ces mêmes forts. Le père Bullet, prêtre missionnaire aumônier au fort français Saint-Louis en 1775, a dessiné deux plans de ce dernier, apportant des détails sur les jardins et les espèces végétales qui s'y trouvaient (Figure 4). Le plan général de Ouidah dressé par Bullet permet de se faire une idée de l'importance de ces jardins par rapport au fort français et à la ville. Les jardins se répartissent en deux zones : de petites parcelles à l'intérieur du fort, et des parcelles de grande taille à l'extérieur, entourées de murs de terre battue mesurant plus de trois cents mètres de long, "rempli[es] d'arbres fruitiers du pays et de l'Amérique ». Ce plan montre une organisation rationnelle de l'espace autour d'allées bordées de fruitiers (Juhé-Beaulaton 1994). On assiste ici à l'arrivée d'un modèle de production basé sur l'ordonnancement de la nature selon des règles strictes, à l'opposé des pratiques locales. Ceci n'a pas empêché les transferts de savoir-faire, et dans les deux sens, car dans cette liste figurent également des plantes locales, comme le tamarin, l'igname et le Synsepalum.

Figure 3 : Forts européens sur la côte du Golfe de Guinée

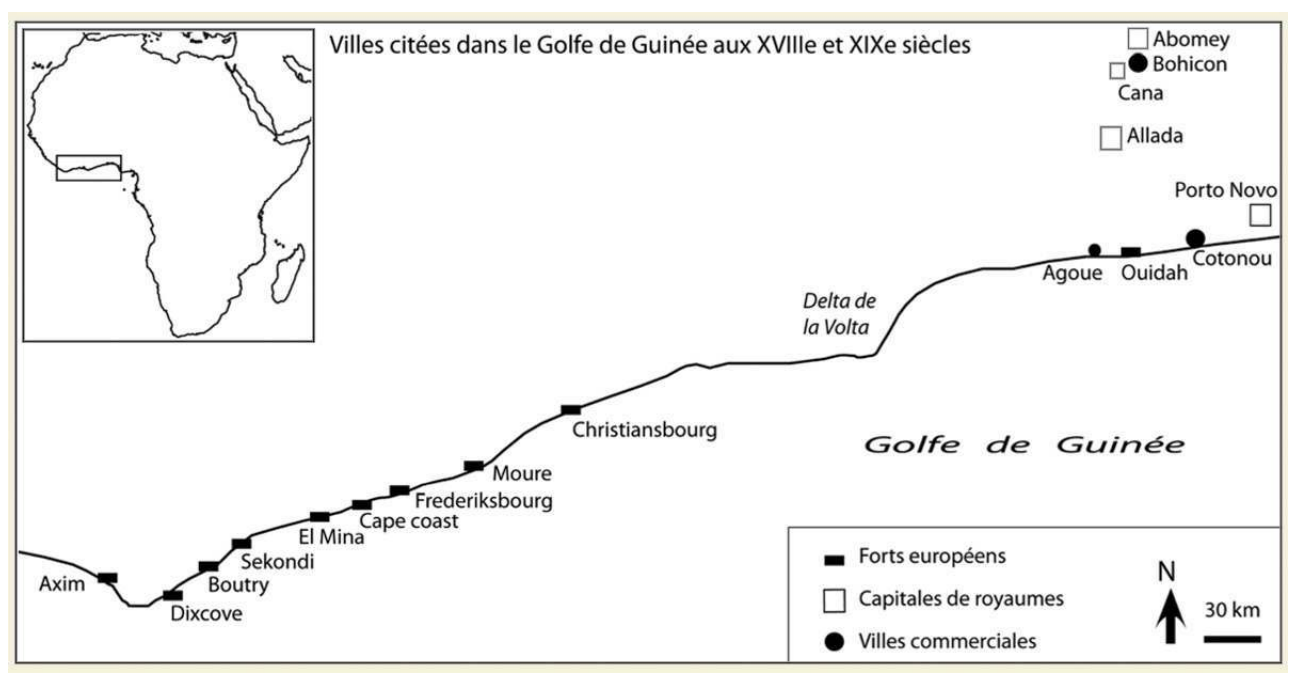




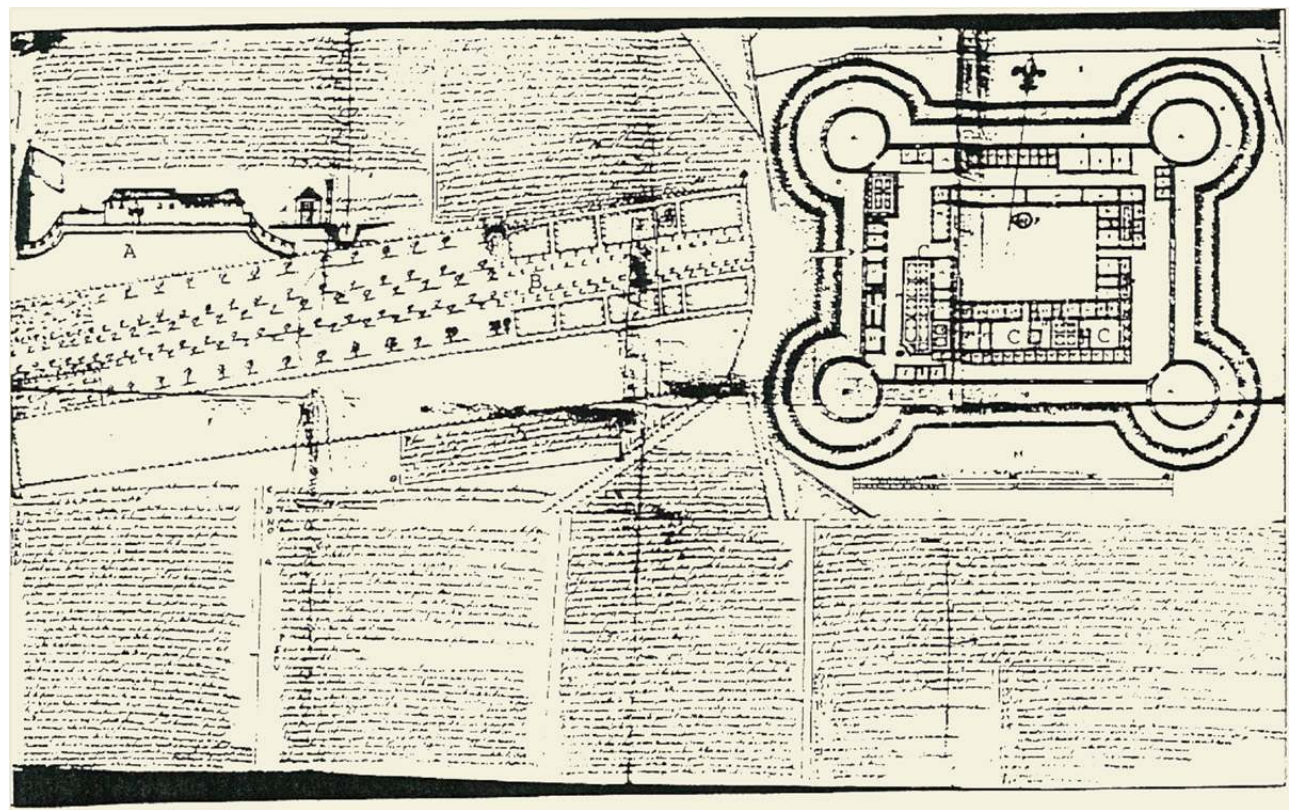

(c) A.N. Paris, Col. C6/27/2

\section{Missions chrétiennes et maraîchage}

20 Avec l'arrivée des missions chrétiennes au XIX ${ }^{\mathrm{e}}$ siècle, entre 1830 et 1860 , les premières fermes-écoles furent créées, souvent associées à des orphelinats ${ }^{14}$. En effet, les missionnaires, dans un souci d'humanité accompagnant leur volonté d'évangélisation, fondèrent les premiers dispensaires médicaux, des écoles et orphelinats où les enfants, d'ailleurs rarement orphelins, étaient éduqués après avoir été isolés de leur famille. Évangélisés, ces derniers devinrent d'ardents défenseurs de la foi chrétienne et les promoteurs d'une nouvelle agriculture plus rationnelle dans l'organisation de sa production, abandonnant les systèmes complexes d'associations végétales utilisées jusqu'alors par les cultivateurs.

21 Les jardins et plantations développés par les missionnaires servirent également de jardins d'essai, comme les jardins des forts avant eux, et ils ont permis d'introduire de " nouvelles ${ }^{15}$ plantes cultivées dont l'intérêt économique prévalait. L'intérêt gustatif et nutritionnel était aussi pris en compte, comme le montre cette lettre du Père Bricet au début $\mathrm{du} \mathrm{xx}^{\mathrm{e}}$ siècle, relatant les actions d'un de ses coreligionnaires, le Père Pichaud, mort au Dahomey en 1902 :

«C'est à lui qu'est due la fondation et l'organisation [...] de cette mission de Zagnanado. L'étranger qui la visite aujourd'hui ne peut se douter de la somme de travail qu'a coûtée au P. Pichaud le défrichement et la plantation de ce coin d'Afrique devenu un Eden enchanteur. Que de produits tropicaux ou européens n'essaya-t-il pas d'acclimater et souvent avec succès. Ses quinze mille cacaoyers, les premiers plantés dans la colonie, sont encore les plus beaux de toute la contrée ; ses arbres à caoutchouc sont magnifiques. Ce fut lui qui importa les bananiers à Zagnanado et même plusieurs espèces inconnues au Dahomey. Il en donnait des régimes et des plants aux chefs du pays qui l'appelaient l'homme aux bananes. Il s'était procuré d'excellentes espèces de mangues qu'on ne trouve pas au Dahomey, 
et ses plantations d'ananas ont un grand renom là-bas. Il entreprit aussi la culture de la vanille. Les directeurs du jardin colonial qu'il avait vus à Paris lui avaient fait bon accueil et lui fournissaient des semences... » (Bricet 1902 : 67). Cotonou (wharf puis port en eau profonde) et Bohicon, toutes deux desservies par une voie ferrée, aux dépens de celles de Ouidah et Abomey. Des jardins d'essais agricoles sont créés à Porto Novo, Niaouli et Abomey pour acclimater des espèces «nouvelles », utiles au développement économique de la colonie. Maïs, riz, arachides, mais aussi café, cacao, coton, tabac... la ville de Porto Novo, ancienne capitale d'un royaume, devint le lieu de résidence du Gouverneur qui y bâtit son palais et son jardin, situés sur l'emplacement d'un sanctuaire boisé lié à l'ancien pouvoir royal. Ce jardin était à la fois un lieu d'agrément et une station d'essais agricoles ${ }^{16}$. Les jardins d'essais établis dans les villes, au Dahomey comme dans les autres colonies, fournissaient des plantes aux Européens et aux cultivateurs locaux via les agents coloniaux qui diffusaient ainsi les savoirs et pratiques du jardinage occidental (Bonneuil 1997). Ce nouvel ordre mis en place sous la contrainte, niant et dénigrant systématiquement les savoirs endogènes, imposant une autre vision de la nature, domestiquée, ordonnée, raisonnée, ne remplaça cependant pas les systèmes locaux; il révéla ses limites rapidement du fait d'une dégradation des sols due aux défrichements excessifs et aux monocultures imposées. Certains agronomes et botanistes, comme Auguste Chevalier (1933), dénoncèrent très tôt ces abus, mais ils ne furent pas entendus. Soulignons que ce modèle d'exploitation agronomique fut élaboré en ville, dans une ville pensée et réaménagée à l'européenne.

\section{La culture dans les villes aujourd'hui}

De nos jours, les villes et villages comportent toujours une végétation arborée relativement dense, où les arbres fruitiers continuent d'occuper une place importante, dans les espaces privés des concessions familiales comme dans les espaces publics ${ }^{17}$. Mais 
la présence d'une production vivrière diffère selon les villes. Dans les villes anciennes, de taille moyenne, même si le tissu bâti s'est densifié, des espèces végétales poussent souvent aux abords des habitations. Ce sont la plupart du temps des plantes utiles, conservées sur place ou plantées, formant une végétation luxuriante en apparence inorganisée, ce qui caractérisait déjà les espaces cultivés en ville avant la colonisation. Le cortège de plantes comprend à la fois des arbres fruitiers, des plantes alimentaires, médicinales, rituelles, des espèces introduites ou indigènes, plantées ou spontanées. Notons que la diversité de la flore s'accroît lorsque le chef de la famille occupe des responsabilités religieuses. Cet espace autour des habitations, clos ou pas, connaît une organisation et une exploitation familiales. C'est aussi un espace de conservation pour certaines plantes utiles qui ne sont plus cultivées en plein champ mais sont plantées à des fins rituelles, comme par exemple d'anciens cotonniers ou certaines ignames.

Bien des villes africaines ont vu apparaître une agriculture maraîchère en périphérie ou dans les zones de bas-fond inondables pour assurer leur approvisionnement en produits potagers (Kakai et al. 2010). Aujourd'hui, des plantes ornementales ont rejoint ce cortège et leur marché est en extension. Ces zones de jardins font l'objet d'une organisation spatiale rigoureuse héritée des pratiques occidentales. Certaines zones considérées comme disponibles dans les villes sont à présent gagnées par le maraîchage dans un contexte d'insécurité foncière. À Cotonou, où la pression démographique et foncière est très forte, certains trottoirs sont mis en culture ainsi que le cordon littoral jusqu'alors domaine de la cocoteraie. Parallèlement à ce maraîchage urbain, une culture domestique, d'apparence inorganisée, fournissant des plantes et des fruits aux habitants, se maintient en ville, voire se développe en relation avec l'insécurité alimentaire grandissante. La production agricole domestique est cependant plus marquée dans les villes anciennes restées à l'écart des circuits commerciaux selon la volonté des autorités coloniales de marginaliser les villes qui avaient eu un rôle politique et économique fort avant la colonisation comme Abomey et Ouidah. 
Figure 5 : Place publique à Abomey

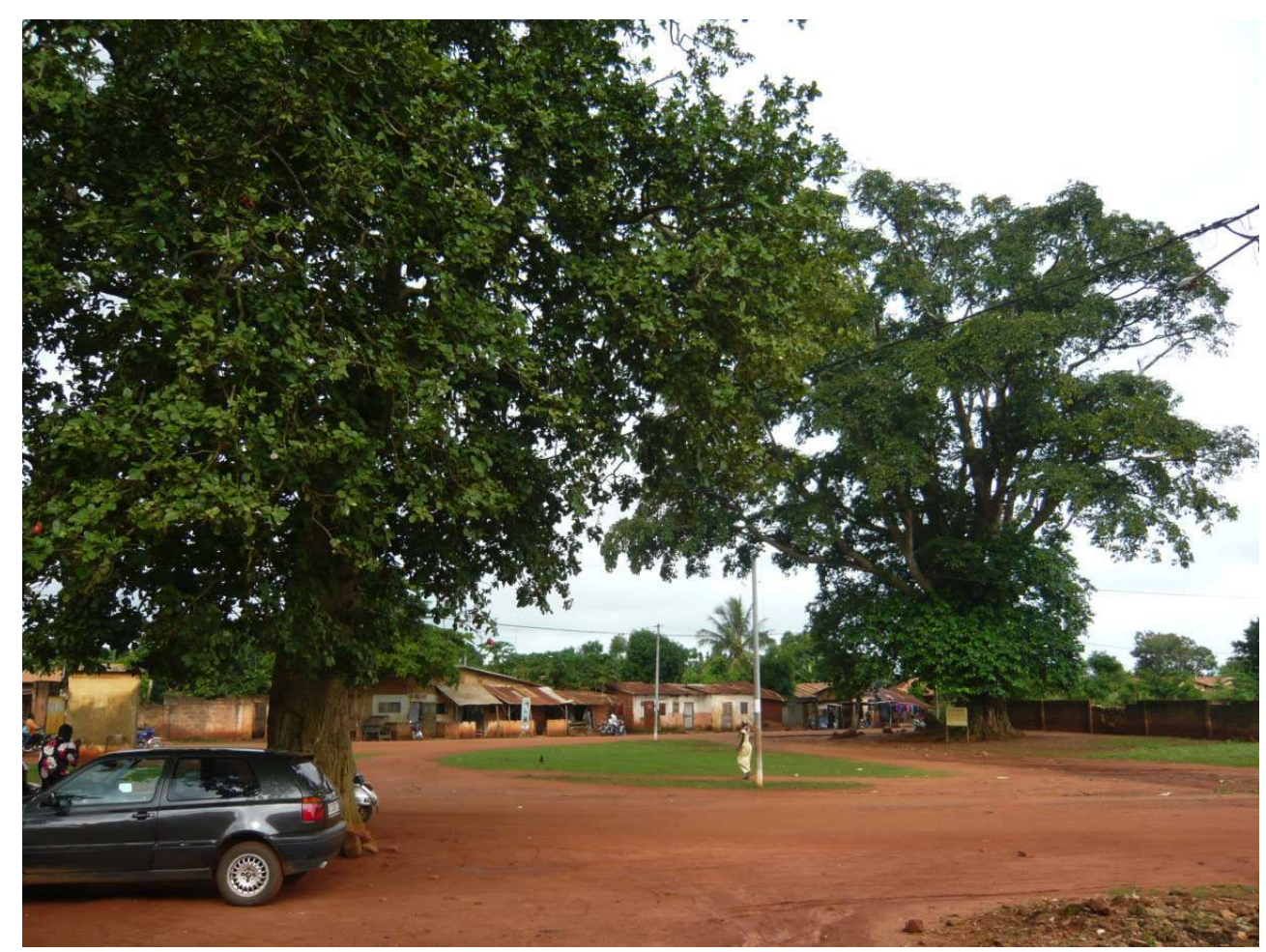

(C) D. Juhé-Beaulaton 2009

\section{Conclusion}

De telles informations invitent à plusieurs commentaires. Notons tout d'abord que les Européens ont longtemps hésité à appeler " villes » les agglomérations dont nous venons de traiter. Ils trouvaient dans le type d'habitat et les techniques architecturales employées - des maisons construites en pisé couvertes de chaume - ainsi que dans la présence de cultures sans organisation apparente, autant de motifs susceptibles de disqualifier des localités au tissu urbain très lâche, et dont l'organisation spatiale semblait encore dominée par les espaces cultivés et arborés. Mais il reste que, avec le temps, la croissance démographique et l'exode rural n'ont pas manqué de contribuer à la densification urbaine et d'entraîner l'insécurité foncière des petits exploitants en ville.

Rappelons ensuite que la multiplicité des usages que les plantes disponibles à proximité des habitations doivent fournir en complément de l'alimentation de base (alimentation, médecine, pratiques magico-religieuses, artisanat) entraîne une certaine diversité végétale, celle-ci restant à étudier. Les espaces habités apparaissent comme des pôles de conservation des plantes anciennement cultivées et des espèces végétales perçues comme rares aujourd'hui dans les espaces non exploités (forêt-brousse), anciens cultivars aujourd'hui abandonnés au profit de variétés introduites ou améliorées. Tel est le cas par exemple de certaines ignames qui ne sont plus cultivées que marginalement dans les enclos des habitations car elles entrent encore dans les offrandes religieuses.

Il est évident enfin que, parce qu'elles se sont toujours imposées, à travers les marchés en particulier, comme des lieux de rencontre et d'échanges entre populations d'origines 
différentes, les villes sont susceptibles de constituer des espaces privilégiés pour les essais agricoles. Mais dans le contexte colonial, le rôle attribué aux missionnaires est venu accentuer cette tendance puisque, alors même que leur mission "première " était d'évangéliser les habitants, il leur était demandé aussi d'«éduquer " ceux-ci en leur faisant adopter un nouveau système d'agriculture basé sur une autre rationalité. Ce système a été renforcé et développé par les administrateurs coloniaux qui ont souvent choisi d'installer eux-mêmes des stations agricoles d'essais dans les villes sous leur juridiction.

Ces vastes espaces habités demeurent ainsi des lieux de production, d'innovation et de conservation agricoles. Les villes continuent de jouer un rôle dans l'adoption de cultures importées, leur acclimatation et leur diffusion dans les systèmes agraires ${ }^{18}$. Ne nous étonnons donc pas si, aujourd'hui, la FAO considère que l'avenir des villes africaines dépend de leur capacité à conserver et développer une production agricole urbaine, encore trop peu considérée par les politiques de développement (FAO 2012).

Je remercie bien sincèrement l'équipe éditoriale du dossier ainsi qu'Anne Luxereau et Dominique Fournier pour leur relecture consciencieuse et leurs remarques constructives pour améliorer ce texte.

\section{BIBLIOGRAPHIE}

Albéca A. L. d' 1895 - La France au Dahomey. Paris, Hachette, 236 p.

Bonneuil C. 1997 - Mettre en ordre et discipliner les tropiques: Les sciences du végétal dans l'empire français, 1870-1940. Paris, Université Paris-Diderot-Paris VII, Thèse de doctorat, 2 vol.

Bosman G. 1705 - Voyage de Guinée : contenant une description nouvelle \& très-exacte de cette côte où l'on trouve \& où l'on trafique l'or, les dents d'elephant, \& les enclaves : de ses pays, royaumes, \& républiques, des mour des habitans, de leur religion, gouvernment, administration de la justice, de leurs guerres, mariages, sepultures, etc ... Utrecht, Chès Antoine Schouten, marchand libraire, 602 p.

Burton R.F. 1864 - A Mission to Gelele, King of Dahomey. London, Tinsley Brothers, 2 vol.

Bricet (père) 1902 - Lettre du père Bricet. L'écho des Missions Africaines de Lyon, Société des Missions Africaines de Lyon, Lyon : 66-73.

Chevalier A. 1933 - Les bois sacrés des Noirs, sanctuaires de la nature. Compte Rendu de la Société de Biogéographie : 37.

Dulucq S. 2004 - Une Afrique de papier. L'imaginaire de la ville dans les colonies françaises. In Dulucq S. et Soubias P. (Ed.) L'espace et ses représentations en Afrique subsaharienne: approches pluridisciplinaires. Paris, Karthala : 203-220.

Fairhead J. \& Leach M. 1996 - Misreading the African Landscape: Society and Ecology in a ForestSavanna Mosaic. Cambridge, Cambridge University Press, 392 p.

FAO 2012 - Pour des villes plus vertes en Afrique. Premier rapport d'étape sur l'horticulture urbaine et périurbaine. Rome. http://www.fao.org/3/a-i3002f.pdf 
Foa E. 1895 - Le Dahomey. Paris, A. Hennuyer, xv-429 p.

Forbes F. E. 1851 - Dahomey and the Dahomans Being the Journals of Two Missions to the King of Dahomey, and Residence at His Capital in the Years 1849 and 1850. London, 2 vol.

Gayibor N.L. 1985 - L'Aire culturelle Ajatado des origines à la fin du XVIII siècle, Paris, Université Paris 1, Thèse de Doctorat d'État, 3 vol.

Harlan J.R. 1987 - Les plantes cultivées et l'homme. Paris, PUF, 446 p.

Hubert H. 1908 - Mission scientifique au Dahomey. Paris, E. Larose, 288 p.

Juhé-Beaulaton D. 1998 - La palmeraie du Sud Bénin avant la colonisation: essai d'analyse historique. In Chastanet M. (Ed.) Plantes et paysages d'Afrique, une histoire à explorer. Paris, Karthala : 327-352.

Juhé-Beaulaton D. 1994 - Les jardins des forts européens de Ouidah (Bénin) : premiers jardins d'essai. Cahiers du Centre de Recherches Africaines $8:$ 84-105.

Kakai H.F., Kakai A.G. \& Tohouegnon A.G. 2010 - Agriculture urbaine et valorisation des déchets au Bénin : une approche de développement durable. Vertigo - la revue électronique en sciences de l'environnement, 2 septembre 2010, 10 (2). En ligne : http://vertigo.revues.org/9994, consulté le 2014-07-23.

Law R. 2004 - Ouidah: The Social History of a West African Slaving Port 1727-1892. Athens, Ohio University Press ; Oxford, James Currey, 320 p.

Le Gleau J.-P., Pumain D. \& Saint-Julien T. 1996 - Villes d'Europe : à chaque pays sa définition. Économie et statistique 294 (1) : 9-23.

Nardin J.-C. 1967 - La reprise des relations franco-dahoméennes au XIX ${ }^{\mathrm{e}}$ siècle : la mission d'Auguste Bouët à la cour d'Abomey (1851). Cahiers d'études africaines 7 (25) : 59-126.

Repin Dr. 1863 - Voyage au Dahomey. Le Tour du monde 161-162-163 : 65-112.

Toutée G. J. 1897 - Dahomé, Niger, Touareg : récit de voyage. Paris, A. Colin, xxii-370 p.

Vallon A., 1861 - Le royaume du Dahomey. Revue maritime et coloniale 2 : 329-363.

Vansina J. 1985 - L'homme, les forêts et le passé en Afrique. Annales : 1307-1334.

\section{ANNEXES}

Liste des plantes citées dans les jardins des forts européens sur la côte ouest africaine ${ }^{19}$ 


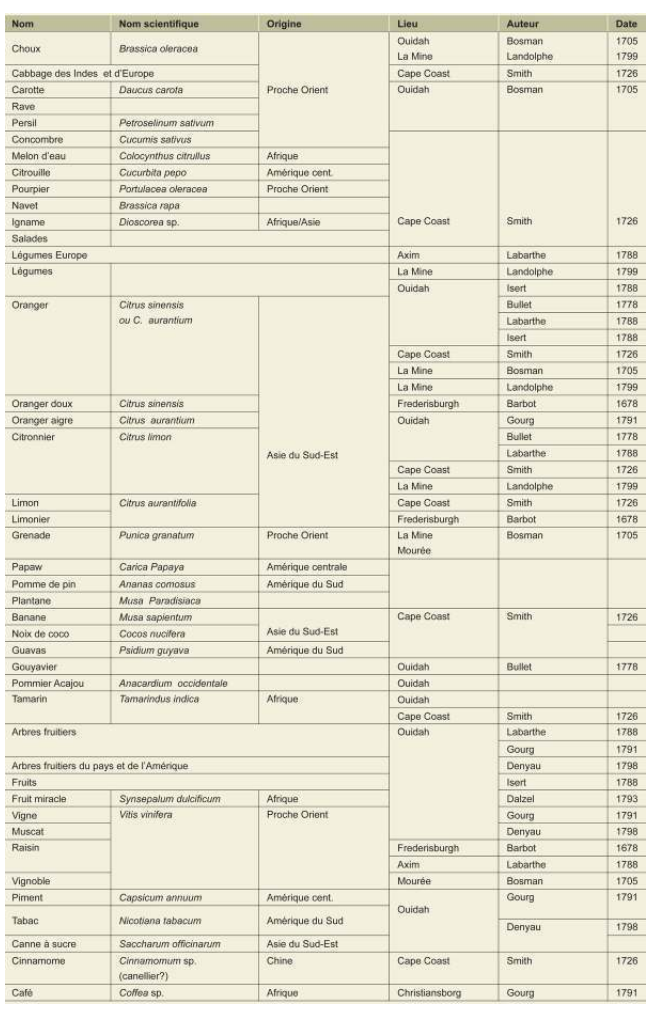

\section{NOTES}

1. Elle est définie par l'existence de traits culturels communs, dont la langue et les pratiques religieuses sont les manifestations principales, s'expliquant en grande partie par une origine géographique commune située à Oyo, une cité yoruba du Nigéria.

2. Ces enquêtes ont d'abord été réalisées dans le cadre de ma thèse de doctorat sur l'histoire des paysages de la région et se sont poursuivies ensuite dans le cadre de mes travaux sur les processus de patrimonialisation.

3. Selon les auteurs, les chiffres varient entre 12000 et 20000 habitants pour Ouidah, 30000 habitants pour Abomey. Ces données démographiques sont basées sur des estimations peu fiables, mais elles donnent une idée de la densité de la population dans ces centres habités. Et si le seuil démographique devait être retenu pour définir la ville, il serait alors largement atteint.

4. La présence de marchés peut également être un critère, même si nombre de villages en disposent aussi. Mais leur hiérarchisation permet de les distinguer entre ceux des villes ou du milieu rural. Le marché Hunjro d'Abomey est aujourd'hui encore un lieu commercial prépondérant : les produits agricoles nouvellement récoltés y sont vendus en premier, après les cérémonies rituelles des prémices.

5. Les capitales royales étaient organisées autour du palais où résidait une population nombreuse composée des épouses du roi qui se comptaient en milliers, de leurs enfants, parentèle, eunuques et esclaves. Chaque roi construisait son propre palais.

6. La fonction religieuse d'Abomey se retrouve dans la ville de Cana située à proximité du lieu d'intronisation des rois.

7. Bouët 1851 : 93 dans Nardin 1967.

8. Le Dahomey mena de nombreuses campagnes militaires contre les royaumes Mahi et Yoruba, au nord et à l'est. 
9. La notion de jardin ou verger telle qu'elle est comprise dans le monde occidental doit être repensée : il s'agit ici souvent d'associations végétales, en apparence inorganisées, sans limites précises.

10. À côté de cette végétation villageoise, figure un certain nombre d'arbres dont la fonction essentielle est de représenter un lieu de commémoration et d'identité communautaire. Il s'agit fréquemment d'irokos, de fromagers, de baobabs.

11. Les noms scientifiques sont précisés dans le texte seulement pour les espèces locales méconnues.

12. Diffusion à mettre en relation avec l'essor du commerce de l'huile vers l'Europe en voie d'industrialisation à la fin du XVIII ${ }^{\mathrm{e}}$ siècle.

13. Voir liste en annexe.

14. Au Dahomey, la première ferme-école a été créée à la mission d'Agoué en 1835 (Albéca 1895 : 164).

15. Pas si nouvelles que cela, au regard de ce qui était déjà cultivé dans les jardins des forts européens.

16. Devenu aujourd'hui le Jardin des Plantes et de la Nature, le jardin d'essai présente au public une collection de plantes économiques et médicinales. On peut y voir aujourd'hui encore les «arbres du gouverneur » plantés alignés, mais aussi les vestiges de l'ancienne forêt sacrée.

17. Voir l'image capturée sur google earth de la ville d'Abomey.

18. Dans le présent numéro, Anne Luxereau mentionne également l'ancienneté de cette horticulture urbaine dont le développement récent montre les capacités d'adaptation des exploitants en réponse non seulement aux contraintes environnementales mais aussi au développement de nouveaux marchés à longue distance.

19. La date indiquée correspond à la date de la première citation et nom son auteur. La bibliographie complète des auteurs figure dans Juhé-Beaulaton (1994) pour ne pas allonger les références bibliographiques de cet article. Les identifications botaniques proposées et les origines sont tirées de Harlan 1987.

\section{RÉSUMÉS}

L'histoire de l'agriculture dans les villes du Golfe de Guinée reste à ce jour peu explorée. Les récits de voyageurs européens et les informations orales recueillies lors d'enquêtes permettent de saisir la place et l'évolution des plantes cultivées en milieu urbain depuis le XVII ${ }^{\mathrm{e}}$ siècle tout en s'interrogeant sur la notion de «ville». À partir d'exemples choisis dans l'ancien royaume du Dahomey (Abomey, Cana, Ouidah dans le sud du Bénin actuel), les sources historiques écrites relatent l'organisation de l'espace habité en distinguant des fonctions différentes selon les villes, autour du commerce ou de l'administration politique tout en révélant une exploitation agricole comparable. Les villes apparaissent à la fois comme des lieux d'innovation agricole et de conservation de certaines plantes. Les premiers contacts avec les Européens (résidents des forts et comptoirs de commerce, missionnaires chrétiens) ont favorisé le développement d'une agriculture maraîchère; l'administration coloniale a ensuite contribué fortement à repenser la ville et l'agriculture par la création de stations agricoles et un nouvel urbanisme. Cependant, les cultures sont toujours restées présentes dans les villes, en périphérie ou dans les espaces interstitiels et les deux «modèles ", le local et l'européen, ont pu coexister sans se concurrencer. 
The history of the agriculture in the cities of the Gulf of Guinea remains this day little explored. The narratives of European travelers and the oral information collected during inquiries allows to know the place and the evolution of plants cultivated in urban zones since the seventeenth century while questioning the notion of « city ». Based on examples from the ancient kingdom of Dahomey (Abomey, Cana, Ouidah in the south of the present Benin), written historical sources tell the organization of space inhabited by distinguishing different functions according to cities around trade or political administration while showing comparable farm. Cities appear at the same time as places of agricultural innovation and preservation of certain plants. The first contacts with the Europeans (residents of forts and trading posts, Christian missionaries) promoted the development of a truck-farming agriculture; then, the colonial administration contributed strongly to rethink the city and agriculture by creating agricultural stations and a new town planning. However, cultures always remained present in cities, in periphery or in the interstitial spaces and both "models", premises(place) and European, could coexist without competing.

\section{INDEX}

Keywords : Africa, Benin, city, history, agriculture, garden

Index géographique : Afrique, Bénin

Mots-clés : ville, histoire, agriculture, jardin

\section{AUTEUR}

DOMINIQUE JUHÉ-BEAULATON

Historienne, UMR 7206 MNHN - CNRS 